\title{
Medication Adherence, Health Care Utilization, and Costs Among Patients Initiating Oral Oncolytics for Multiple Myeloma or Chronic Lymphocytic Leukemia/Small Lymphocytic Lymphoma
}

\author{
Ankur A. Dashputre, MS; Katie S. Gatwood, PharmD; and Justin Gatwood, PhD
}

\begin{abstract}
BACKGROUND: Oral oncolytic therapies have improved survival in hematologic cancers, such as chronic lymphocytic leukemia/small lymphocytic lymphoma (CLL/SLL) and multiple myeloma (MM), which are now being managed like chronic conditions. However, compared with other cancers, there is a lack of studies assessing adherence, health care resource utilization, and costs in patients with these cancers.

OBJECTIVE: To assess factors associated with adherence to oral oncolytic therapies, health care utilization, and costs in patients with CLL/SLL or MM.

METHODS: A retrospective database study was conducted using the IBM MarketScan Commercial Claims and Medicare Supplement databases. Adults (aged $\geq 18$ years) diagnosed with and prescribed an oral oncolytic for CLL/SLL (ibrutinib or idelalisib) or MM (thalidomide, lenalidomide, or pomalidomide) between 2013 and 2016 and with continuous eligibility 6 months before and 12 months after oral oncolytic initiation were identified. Adherence to oral oncolytics was measured using the proportion of days covered (PDC) metric. Multiple linear regression and multivariable logistic regression were used to identify adherence predictors. Count models assessed the relationship between adherence and resource utilization, and generalized linear models assessed the relationship between adherence and health care costs.
\end{abstract}

RESULTS: A total of 701 and 2,385 patients were identified with CLL/SLL or MM, respectively. Mean PDC (SD) for CLL/SLL and MM patients was 75.3 (22.5) and 57.6 (26.5), respectively. For CLL/SLL patients, those aged $\geq 65$ years (beta $[B]=-4.00$ ) had lower medication use. Among MM patients, multiple predictors of higher medication use emerged: aged $\geq 65$ years $(B=3.44)$, higher than average outpatient resource utilization $(B=3.53)$, insurance plan other than preferred provider organization (PPO; $B=-2.58$ ), previous cancer therapy $(B=-2.81)$, higher number of concurrent unique therapeutic classes $(B=-0.35)$, and higher comorbidity burden $(B=-2.55)$. Patients with CLL/SLL and enrolled in plans other than a PPO were more likely to be adherent $(O R=1.41,95 \% \mathrm{Cl}=1.01-1.98)$, whereas patients who were aged $\geq 65$ years, were residents of the southern United States, and had visited the emergency department in the baseline period were less likely to be adherent. For MM patients, those aged $\geq 65$ years $(0 R=1.68$, $95 \% \mathrm{Cl}=1.38-2.04)$ and with higher than average outpatient services utilization $(0 \mathrm{R}=1.24,95 \% \mathrm{Cl}=1.01-1.52)$ were more likely to be adherent, whereas those enrolled in plans other than a PP0, previously treated with cancer therapy, and with higher comorbidity burden were less likely to be adherent. In both cohorts, adherent patients had significantly lower odds of health care utilization and incurred lower medical costs, but higher prescription costs, following oncolytic initiation; however, total costs were not significantly lower in those adherent.

CONCLUSIONS: Factors were identified that influenced adherence at the patient, treatment, and health system levels. These factors can be used to identify patients requiring interventions for improving medication-taking behavior and associated health care burden.

J Manag Care Spec Pharm. 2020;26(2):186-96

Copyright $\odot 2020$, Academy of Managed Care Pharmacy. All rights reserved.

\section{What is already known about this subject}

Treatment paradigms have changed in chronic lymphocytic leukemia/small lymphocytic lymphoma (CLL/SLL) and multiple myeloma (MM) following approval of novel oral oncolytics.

Adherence to oral oncolytics is critical to successful cancer outcomes, and poor adherence to oral oncolytics has been consistently identified as a driver of increased health care burden and costs and poor survival.

Research is limited on adherence to oral oncolytics for MM and CLL/SLL and its effect on health care utilization and costs.

\section{What this study adds}

This study identified factors associated with medication adherence and the effect of medication adherence on health care utilization and costs in CLL/SLL or MM, an area that has been understudied in these cancers.

Patient, treatment, and health system factors, such as older age, increased medication and comorbidity burden, previous cancer therapy, health insurance type, and higher outpatient visits, were identified as factors influencing adherence to oral therapies for CLL/SLL or MM.

Adherent patients had a significantly lower risk of health care utilization and incurred lower medical but higher prescription costs.

$\mathrm{M}$ ultiple myeloma (MM) and chronic lymphocytic leukemia/small lymphocytic lymphoma (CLL/SLL) are hematologic cancers that affect the plasma cells and lymphocytes, respectively., ${ }^{1,2}$ Both cancers are commonly diagnosed between ages 65 and 74 years, slightly predominant in males, with MM being more common in blacks, and CLL/SLL being more common in whites. ${ }^{3}$ As of 2016, the current incidence of CLL/SLL and MM are 4.9 and 6.9 per 100,000 cases with a 5 -year survival rate of $84.2 \%$ and $50.7 \%$, respectively. ${ }^{3}$ 


\section{Medication Adherence, Health Care Utilization, and Costs Among Patients Initiating Oral Oncolytics for Multiple Myeloma or Chronic Lymphocytic Leukemia/Small Lymphocytic Lymphoma}

The introduction of novel targeted oral therapies such as lenalidomide, pomalidomide, panobinostat, and ixazomib for MM and ibrutinib and idelalisib for CLL/SLL has changed the treatment paradigm for these malignancies. These oral targeted agents have contributed significantly to increased survival in addition to improving the ease of administration. ${ }^{4-7}$ The improved survival benefit offered by oral therapies has changed the treatment strategies for these malignancies, which are now more commonly managed as chronic conditions. Consequently, the chronic management strategy and mode of drug administration have shifted the burden of treatment and adherence to the patient. Similar to chronic conditions, nonadherence to oral oncolytics is associated with cancer progression and worse survival outcomes. ${ }^{8}$ Additionally, nonadherence to oral therapies also leads to increased health care utilization and costs. ${ }^{8,9}$ Consequently, adherence to oral oncolytics and identification of key drivers (e.g., patient, health care, environmental factors) of medication use are critical to the success of cancer management and devising adherence-promoting strategies to achieve desirable patient outcomes. For example, numerous studies in chronic myeloid leukemia (CML), another type of hematologic cancer mainly treated by oral therapies (tyrosine kinase inhibitors), have identified age, concurrent medications, comorbidity burden, cost sharing, and insurance subsidy as some of the drivers of adherence, with adherence associated with lower health care utilization, lower costs, and better survival. ${ }^{10-18}$ Similarly, it is critical to study and identify drivers of adherence and associated outcomes in patients with MM or CLL/SLL initiating oral oncolytics.

Although some studies have assessed medication adherence, health care utilization, and costs in MM, there is a dearth of real-world studies assessing factors associated with adherence to oral therapies, health care utilization, and costs in MM and CLL/SLL. The studies on MM therapies focused on treatment patterns and adherence, ${ }^{19-21}$ health care utilization and costs, ${ }^{22}$ and association of cost-sharing and adherence ${ }^{23}$; however, these studies did not assess factors associated with adherence and the association of adherence with health care utilization and costs.

Specifically, in MM, adherence to oral therapies can be burdensome to the patient due to the complex treatment regimens and associated high therapy costs, side effects, and variable dosing patterns for oncolytics within a treatment regimen, making identifying factors influencing adherence even more critical. ${ }^{24-26}$ Considering the current gaps in understanding medication use in MM and CLL/SLL, this study sought to determine factors associated with adherence to oral therapies, and the association of adherence with health care utilization and costs for patients with MM or CLL/SLL initiating oral oncolytics.

\section{Methods}

\section{Data Source}

This study used data from the January 1, 2013-December 31, 2016 IBM MarketScan Commercial Claims and Medicare Supplement databases. These databases provide a nationally representative convenience sample of individuals (and their dependents) covered by employer-sponsored or Medicare supplemental health insurance, which include patient demographics, enrollment information, and adjudicated claims for health care experiences (outpatient medical, inpatient medical, and outpatient prescription drugs) across the continuum of care. The databases are deidentified and fully compliant with the Health Insurance Portability and Accountability Act of 1996. This study was determined to be exempt by the University of Tennessee Health Science Center Institutional Review Board.

\section{Patient Selection}

Patients were included in the analysis if they met the following criteria for study years 2013 through 2016: (a) at least 1 diagnosis claim using the International Classification of Diseases, Ninth Revision, Clinical Modification (ICD-9-CM before October 2015) and ICD-10-CM (from October 2015) for MM (ICD9-CM: 203.0x; ICD-10-CM: C90.0x) or CLL/SLL (ICD-9-CM: 204.1x, 200.8x; ICD-10-CM: C91.1x, C83.0x); (b) at least 2 prescription claims for immunomodulating agents (thalidomide, lenalidomide, or pomalidomide) for MM or any kinase inhibitor (ibrutinib or idelalisib) for CLL/SLL; (c) continuous eligibility 6 months pre-index (baseline) through 12 months post-index (follow-up); and (d) at least aged 18 years at index date, which was defined as the date of first prescription for selected oncolytic agents (Appendix A, available in online article). Additionally, as an exploratory supplementary analysis, patients prescribed panobinostat (a histone deacetylase inhibitor) or ixazomib (a proteasome inhibitor) for MM were included. However, for these agents, a shorter follow-up period of 6 months was chosen as they were approved in 2015.

\section{Patient Characteristics}

Patient demographics extracted at index included age, sex, region of residence (Northeast, North Central, South, West), urbanicity (metropolitan statistical area [MSA] or non-MSA), and health plan type and primary payer. Comorbidity burden was measured using the National Cancer Institute Charlson Comorbidity Index ( $\mathrm{NCI} C \mathrm{CI}$ ), calculated based on contributing comorbidities in the baseline period, excluding cancer. ${ }^{27}$ Index prescription characteristics included fill location and patient cost sharing, calculated as the sum of reported coinsurance and copay amounts. Other characteristics measured during the baseline period included counts of outpatient medical, inpatient medical, emergency department visits, the number of concurrent unique therapeutic classes, and receipt of cancer therapy. 


\begin{tabular}{|c|c|c|}
\hline \multirow{2}{*}{$\begin{array}{l}\text { Characteristic } \\
\text { Age at index, years, mean (SD) }\end{array}$} & $\begin{array}{l}\text { CLL/SLLa } \\
(\mathrm{n}=701)\end{array}$ & $\begin{array}{c}\mathrm{MM}^{\mathrm{b}} \\
(\mathrm{n}=2,385)\end{array}$ \\
\hline & $67.1(11.5)$ & $63.5(11.1)$ \\
\hline \multicolumn{3}{|l|}{ Age group at index, years, $\mathrm{n}(\%)$} \\
\hline $18-64$ & $348(49.6)$ & $1,412(59.2)$ \\
\hline$\geq 65$ & $353(50.4)$ & $973(40.8)$ \\
\hline \multicolumn{3}{|l|}{ Sex, n (\%) } \\
\hline Male & $456(65.0)$ & $1,333(55.9)$ \\
\hline Female & $245(35.0)$ & $1,052 \quad(44.1)$ \\
\hline \multicolumn{3}{|l|}{ Region, $\mathrm{n}(\%)^{\mathrm{c}}$} \\
\hline Northeast & $188(26.8)$ & $584(24.5)$ \\
\hline North Central & $158(22.5)$ & $538(22.6)$ \\
\hline South & $260(37.1)$ & $977(40.9)$ \\
\hline West & $95(13.5)$ & $260(10.9)$ \\
\hline Metropolitan statistical area, n (\%) & $600(85.6)$ & $2,036(85.3)$ \\
\hline \multicolumn{3}{|l|}{ Benefit plan, $\mathrm{n}(\%)^{\mathrm{c}}$} \\
\hline Comprehensive & $133(18.9)$ & $385(16.1)$ \\
\hline EPO/HMO & $83(11.8)$ & $265(11.1)$ \\
\hline POS/POS with capitation & $46 \quad(6.6)$ & $173 \quad(7.2)$ \\
\hline $\mathrm{PPO}$ & $362(51.6)$ & $1,321 \quad(55.4)$ \\
\hline CDHP/HDHP & $66 \quad(9.4)$ & $214 \quad(8.9)$ \\
\hline $\begin{array}{l}\text { Cancer therapy in baseline } \\
\text { period, } \mathrm{n}(\%)^{\mathrm{d}}\end{array}$ & $317(45.2)$ & $1,584(66.4)$ \\
\hline $\begin{array}{l}\text { Unique therapeutic classes in } \\
\text { baseline period, mean (SD) }\end{array}$ & $7.4 \quad(4.8)$ & $8.5 \quad(4.6)$ \\
\hline \multicolumn{3}{|l|}{ Index prescription place, $\mathrm{n}(\%) \mathrm{c}$} \\
\hline Retail & $628(89.6)$ & $1,737(72.8)$ \\
\hline Mail order & $5 \quad(0.7)$ & $561(23.5)$ \\
\hline \multicolumn{3}{|l|}{ Index copay, n (\%) } \\
\hline$\$ 0$ & $162(23.1)$ & $523(21.9)$ \\
\hline$\$ 1-\$ 50$ & $261(37.2)$ & $1,056(44.3)$ \\
\hline$\$ 51-\$ 100$ & $127(18.1)$ & $432(18.1)$ \\
\hline$>\$ 100$ & $151(21.5)$ & $374(15.7)$ \\
\hline $\begin{array}{l}\text { Baseline outpatient visits, } \\
\text { mean (SD) }\end{array}$ & $17.8(13.1)$ & $22.3(13.1)$ \\
\hline Baseline inpatient visits, mean (SD) & $0.3 \quad(0.8)$ & $0.4 \quad(0.7)$ \\
\hline $\begin{array}{l}\text { Baseline emergency room visits, } \\
\text { mean (SD) }\end{array}$ & $0.3 \quad(0.8)$ & $0.3 \quad(0.8)$ \\
\hline $\begin{array}{l}\text { NCI Charlson Comorbidity Index, } \\
\text { mean (SD) }\end{array}$ & $0.4 \quad(0.8)$ & $0.7 \quad(1.1)$ \\
\hline \multicolumn{3}{|l|}{ Primary payer, $\mathrm{n}(\%)$} \\
\hline Commercial & $346(49.4)$ & $1,401 \quad(58.7)$ \\
\hline Medicare & $355(50.6)$ & $984 \quad(41.3)$ \\
\hline \multicolumn{3}{|c|}{ 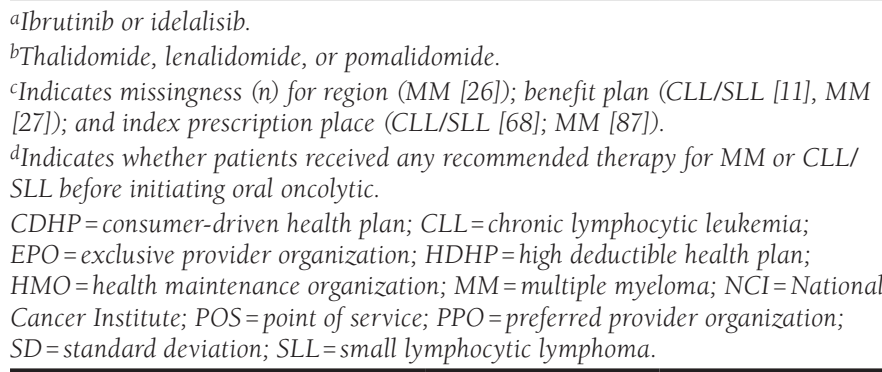 } \\
\hline
\end{tabular}

\section{Adherence}

The proportion of days covered (PDC) metric measured adherence to oral oncolytics and was calculated as a percentage $(0 \%$ $100 \%$ ) of the ratio of the sum of days supplied to the number of days of follow-up (365 days). ${ }^{28}$ Additionally, we also assessed adherence over 90 days, 180 days, and 270 days. Patients with a PDC $\geq 80 \%$ were considered adherent. As an established, acceptable adherence threshold does not exist for hematologic cancers, $\mathrm{PDC} \geq 85 \%$ and $\mathrm{PDC} \geq 90 \%$ as adherent were used as sensitivity analysis. The adherence threshold (medication possession ratio [MPR] or PDC) of $\geq 85 \%$ has been previously used in claims-based studies assessing adherence to therapies for chronic myeloid leukemia. ${ }^{29-32}$ A fixed follow-up of 180 days was used to assess adherence to panobinostat and ixazomib.

\section{Health Care Utilization and Costs}

All-cause health care utilization was evaluated for outpatient, inpatient, and emergency department visits in the follow-up period. Costs associated with use were reported as medical costs (sum of costs for each visit type), and those associated with outpatient pharmacy utilization were reported as pharmacy costs. All costs were inflated to 2019 dollars using the medical care component of the Bureau of Labor Statistics Consumer Price Index. ${ }^{33}$

\section{Statistical Analysis}

Descriptive statistics were used for patient characteristics with means (standard deviation [SD]) reported for continuous variables and counts (plus proportions) reported for categorical variables. Multiple linear regression and multivariable logistic regression were used to identify factors associated with adherence as a continuous measure and binary measure, respectively. Logistic regression models used both the base case PDC $\geq 80 \%$ threshold and the sensitivity thresholds (PDC $\geq 85 \%$ and PDC $\geq 90 \%$ ). Negative binomial regression assessed association of adherence with outpatient visits, whereas inpatient and emergency department visits were collapsed together, and zeroinflated Poisson regression was used for these resource utilization categories. Multivariable generalized linear models with a gamma distribution and log link assessed the association of adherence to oncolytic use with total medical and pharmacy costs, separately and as total costs (total medical cost + prescription cost). All multivariable models were adjusted for baseline characteristics such as age; sex; region of residence; urbanicity; health plan type; NCI CCI; index prescription fill location; index copay; baseline outpatient, inpatient, and emergency department visits; number of concurrent unique therapeutic classes; and prior receipt of cancer therapy. Index prescription fill location was excluded in the models for CLL/SLL due to significantly low numbers in the mail order category. A 2-sided $P$ value of $<0.05$ was considered statistically significant, and all 
Medication Adherence, Health Care Utilization, and Costs Among Patients Initiating Oral Oncolytics for Multiple Myeloma or Chronic Lymphocytic Leukemia/Small Lymphocytic Lymphoma

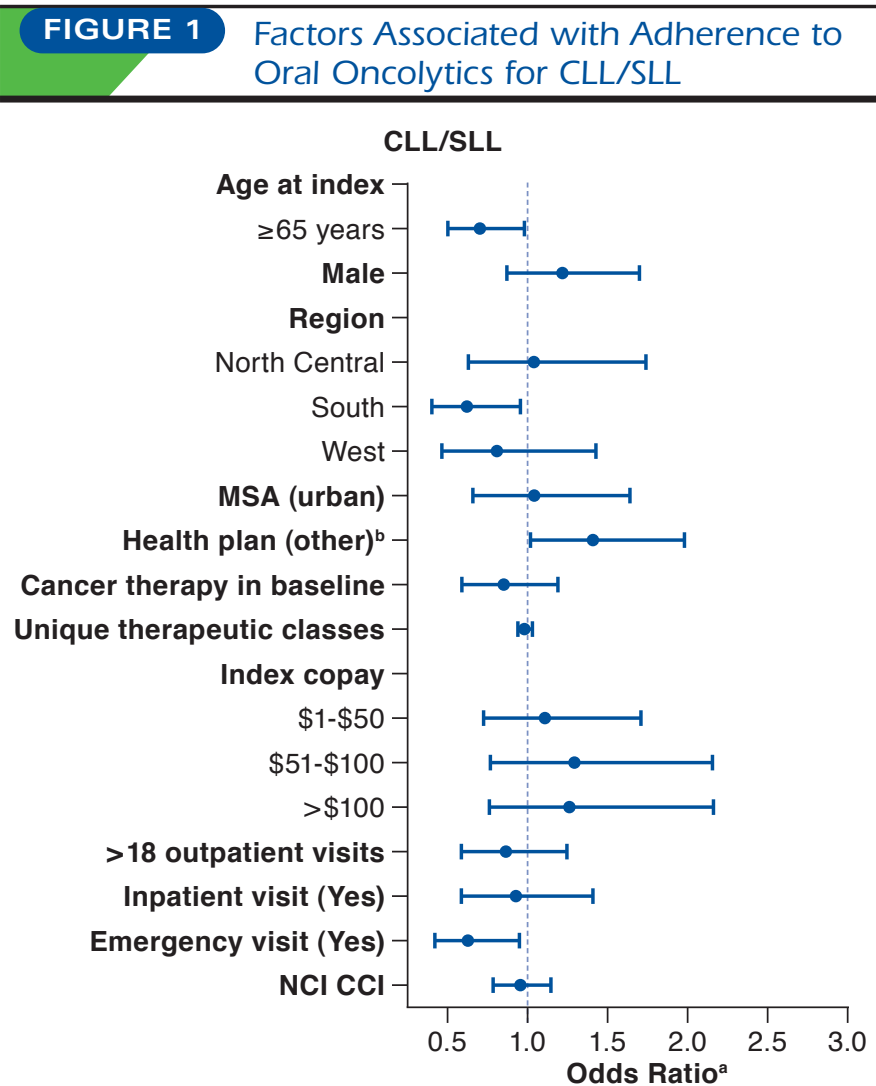

aAdjusted odds ratio estimated by multivariable logistic regression for assessing factors associated with adherence (PDC $\geq 80 \%$ ) to oral oncolytics for CLL/SLL.

${ }^{b}$ Other category includes comprehensive, consumer-directed health plan, exclusive provider organization, health maintenance organization, high-deductible health plan, point of service with/without capitation.

$C C I=$ Charlson Comorbidity Index; $C L L=$ chronic lymphocytic leukemia;

MSA = metropolitan statistical area; $N C I=$ National Cancer Institute; $S L L=$ small lymphocytic lymphoma.

was negatively associated with adherence among CLL/SLL patients. Among MM patients, being aged 65 years and older $(B=3.44,95 \% C I=1.13,5.76)$ and having higher than average (>22 visits) outpatient utilization ( $\mathrm{B}=3.53,95 \% \mathrm{CI}=1.12-5.94$ ) was positively associated with adherence, whereas being on a benefit plan other than a $\mathrm{PPO}(\mathrm{B}=-2.58,95 \% \mathrm{CI}=-4.86$, $-0.31)$, previous cancer therapy $(B=-2.81,95 \% C I=-5.28$, -0.35 ), a higher number of concurrent unique therapeutic classes ( $B=-0.35,95 \% C I=-0.63,-0.08)$, and higher NCI CCI $(B=-2.55,95 \% C I=-3.61,-1.49)$ were negatively associated with adherence (Table 2).

Using a $\mathrm{PDC} \geq 80 \%$, patients with CLL/SLL who were aged 65 years and older (vs. aged 18-64 years), resided in the southern United States (vs. the Northeast), and had emergency department visits in the baseline period were less likely to be adherent. Whereas, those enrolled in plans other than a PPO

\section{FIGURE 2 Factors Associated with Adherence to} Oral Oncolytics for Multiple Myeloma

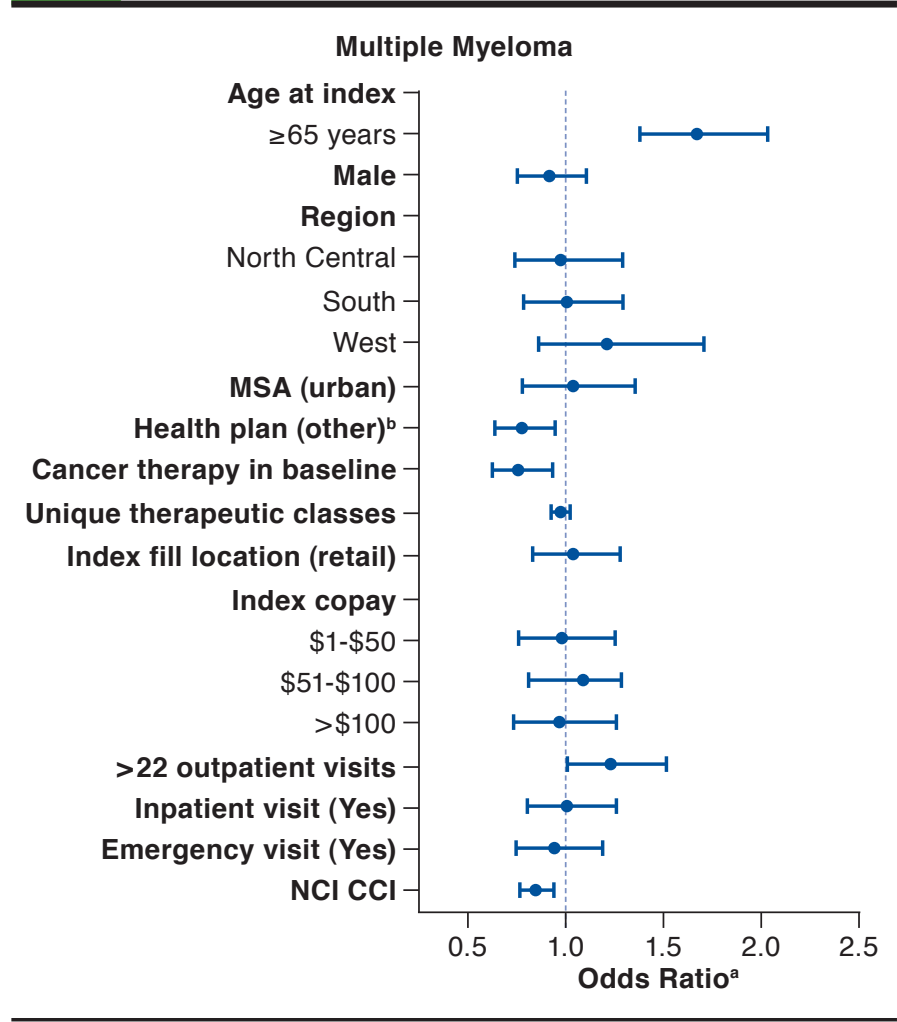

a Adjusted odds ratio estimated by multivariable logistic regression for assessing factors associated with adherence (PDC $\geq 80 \%)$ to oral oncolytics for multiple myeloma.

bOther category includes comprehensive, consumer-directed health plan, exclusive provider organization, health maintenance organization, high-deductible health plan, point of service with/without capitation.

$C C I=$ Charlson Comorbidity Index; CLL = chronic lymphocytic leukemia; MSA = metropolitan statistical area; $N C I=$ National Cancer Institute;

$P D C=$ proportion of days covered; SLL = small lymphocytic lymphoma.

were more likely to be adherent (Figure 1). However, for the $\mathrm{PDC} \geq 85 \%$ threshold, only residence in the southern United States was associated with lower adherence, whereas no factor was significantly associated with the PDC $\geq 90 \%$ threshold (Appendix B, available in online article). For MM patients, the logistic regression using a $\mathrm{PDC} \geq 80 \%$ revealed that those aged 65 years and older (vs. aged 18-64 years) and with higher than average (> 22 visits) outpatient utilization were more likely to be adherent, whereas those enrolled in a plan other than a PPO, previously treated with cancer therapy, and with a higher $\mathrm{NCI}$ CCI were less likely to be adherent (Figure 2). The sensitivity models using the PDC $\geq 85 \%$ and $\geq 90 \%$ thresholds for MM showed similar patterns with those aged 65 years and older and having a higher than average ( $>22$ visits) outpatient utilization having higher odds of adherence, whereas those covered by insurance other than a PPO and with higher NCI CCI 


\section{Medication Adherence, Health Care Utilization, and Costs Among Patients Initiating Oral Oncolytics for Multiple Myeloma or Chronic Lymphocytic Leukemia/Small Lymphocytic Lymphoma}

TABLE 3 Association of Adherence to Oral Oncolytics with Health Care Utilization and Costs

\begin{tabular}{|c|c|c|c|c|}
\hline & \multicolumn{2}{|c|}{ CLL/SLL ${ }^{a}$} & \multicolumn{2}{|c|}{$\mathrm{MM}^{\mathrm{b}}$} \\
\hline & Adherent & Nonadherent & Adherent & Nonadherent \\
\hline \multicolumn{5}{|l|}{ Health care utilization ${ }^{c}$} \\
\hline Outpatient use $\mathrm{d}^{\mathrm{d}}$ & $0.80(0.75-0.86)$ & Reference & $0.78 \quad(0.74-0.81)$ & Reference \\
\hline Inpatient/emergency use ${ }^{\mathrm{d}}$ & $0.73(0.62-0.87)$ & Reference & $0.64(0.57-0.72)$ & Reference \\
\hline \multicolumn{5}{|l|}{ Health care costs ${ }^{\mathrm{e}}$} \\
\hline Total medical costs $(\$)^{\mathrm{d}, \mathrm{f}}$ & 55,141 & 127,198 & 81,762 & 191,541 \\
\hline Prescription costs $(\$)^{\mathrm{d}}$ & 128,561 & 74,627 & 151,442 & 79,769 \\
\hline \multicolumn{5}{|c|}{$\begin{array}{l}\text { aIbrutinib or idelalisib. } \\
\text { 'Thalidomide, lenalidomide, or pomalidomide. } \\
\text { 'Adjusted incidence rate ratios and } 95 \% \text { CI for the association between adherence (PDC } \geq 80 \% \text { ) and outpatient utilization (negative binomial regression) and inpatient/ } \\
\text { emergency utilization (zero-inflated Poisson regression), adjusted for age; sex; region of residence; urbanicity; health plan type; NCI CCI; index prescription fill location } \\
\text { (MM model only); index copay; baseline outpatient, inpatient, and emergency department visits; number of concurrent unique therapeutic classes; and prior receipt of } \\
\text { cancer therapy. } \\
\text { dP<0.05. } \\
\text { eAdjusted costs estimated by multivariable generalized linear models with a gamma distribution and log link adjusted for age; sex; region of residence; urbanicity; health } \\
\text { plan type; NCI CCI; index prescription fill location (MM model only); index copay; baseline outpatient, inpatient, and emergency department visits; number of concurrent } \\
\text { unique therapeutic classes; and prior receipt of cancer therapy. }\end{array}$} \\
\hline
\end{tabular}

having lower odds of adherence. Additionally, for the PDC $\geq 85 \%$ threshold, a greater number of unique concurrent therapeutic classes in the baseline was associated with lower adherence odds (Appendix B).

Adherence was associated with a reduced risk of all-cause health care utilization for both cohorts. Among CLL/SLL patients, those adherent (PDC $\geq 80 \%$ ) to oncolytic therapy had lower outpatient utilization (incidence rate ratio [IRR] $=0.80$, 95\% CI $=0.75-0.86)$ and inpatient/emergency utilization (IRR $=0.73,95 \% \mathrm{CI}=0.62-0.87$ ). Similarly, in the MM cohort, being adherent was associated with lower risk of outpatient (IRR $=0.78,95 \% \mathrm{CI}=0.74-0.81)$ and inpatient $(\mathrm{IRR}=0.64$, $95 \% \mathrm{CI}=0.57-0.72)$ visits. Accordingly, those adherent to oral oncolytics incurred significantly lower all-cause total medical costs. However, prescription costs were significantly higher among adherent patients (Table 3). Total costs (medical + prescription) were not significantly lower for CLL/SLL or MM patients. Adjustments to the threshold for adherence did not yield appreciable differences in results.

\section{Discussion}

Using administrative claims from the IBM MarketScan Commercial Claims and Medicare Supplement databases, we examined factors associated with medication adherence among patients with CLL/SLL or MM initiating oral oncolytic therapy. Our results highlight that adherence to oral oncolytics is higher over the initial period after initiating oral oncolytic but reduces over time. Furthermore, we used these data to relate adhering to oral oncolytics with health care utilization and costs. For CLL/SLL patients, we found that being enrolled in a plan other than a PPO was associated with higher odds of adherence; conversely older age, residing in the southern United States, emergency department visits before oncolytic initiation were associated with a lower odds of adherence. Among patients with MM, older age and higher outpatient utilization were associated with an increased likelihood of being adherent, whereas being enrolled in a plan other than a PPO, previous cancer treatment, and higher comorbidity burden were associated with a lower likelihood of adherence. Being adherent was also associated with a reduced risk of all-cause health care utilization, lower overall medical costs, and higher prescription costs compared with those less adherent to oral oncolytic therapy.

Compared with existing research on MM, we observed comparatively lower adherence rates to the immunomodulating agents thalidomide, lenalidomide, and pomalidomide. Using specialty pharmacy data, Lee et al. (2016) observed an MPR of $83 \%$, compared with a PDC of $58 \%$ in the current analysis. ${ }^{23}$ The lower adherence in our study may be attributable to the difference in study population and measure used for adherence assessment (MPR double counts prescription fills) and may inflate the measured adherence level. ${ }^{34}$ Similarly, for our exploratory assessment of adherence to panobinostat, we observed lower adherence (PDC 52\%) compared with adherence (MPR 90\%) observed by Chari et al. (2018). ${ }^{19}$ In our study, adherence to oral therapies for MM ranged from 52\% to $71 \%$, whereas higher adherence was observed for the CLL/ SLL patients. A systematic review by Greer et al. (2016) found that adherence to oral oncolytics ranged from $46 \%$ to $100 \%$ depending on the type of cancer, adherence measure, patient sample, medication type, and follow-up period. ${ }^{8}$ Although our 


\section{Medication Adherence, Health Care Utilization, and Costs Among Patients Initiating Oral Oncolytics for Multiple Myeloma or Chronic Lymphocytic Leukemia/Small Lymphocytic Lymphoma}

estimates for adherence lie within the upper and lower estimates in the systematic review, it can be said that adherence to the selected agents for MM and CLL/SLL was suboptimal, especially for the MM agents.

Ruddy et al. (2009) proposed a biophysical model that identified personal factors, treatment factors, and interaction with the health care system as potential influencers on medication adherence and persistence. ${ }^{35}$ In the context of this model, we identified that personal factors such as age, residence, and comorbidity burden; treatment factors, such as exposure to previous cancer therapies and burden of multiple medications; and system factors, such as a greater number of outpatient visits, contributed to adherence. Systematic reviews reported mixed results on the influence of age (older vs. younger), geographic area of residence, comorbidity burden (higher vs. lower), medication burden, exposure to previous cancer therapies, and health care utilization as facilitators/barriers for adherence to oral oncolytics. ${ }^{8,35,36}$ For example, there is no consensus on whether factors such as age or comorbidity burden positively or negatively affect adherence to oral oncolytics. ${ }^{8,36}$ Patients with CLL/SLL and MM are more likely to be diagnosed around age 70 years, thus it is critical for older patients with these cancers to be adherent to their oral therapies to get the survival benefit offered by these agents. ${ }^{3}$

Although adherence in comparatively younger adults with these malignancies is as important as in older adults, older adults might be more likely to suffer from multimorbidity and thus require more specialized disease management. ${ }^{37}$ An increased comorbidity and therapy burden are known to negatively affect oral medication adherence. ${ }^{38-41}$ Specifically, for patients with MM, it is critical to be adherent to not only their oral therapies but also their entire regimen, given the complex regimens for MM. ${ }^{24}$ We observed a negative association between previous exposure to MM-specific cancer therapies and adherence to MM oral oncolytics. Management of MM typically includes multiple therapeutic agents ( 2 or more) such as targeted therapies, chemotherapy, or corticosteroids that can be administered by different routes, thus increasing regimen complexity, a treatment factor affecting medication adherence. ${ }^{24,42,43}$

We also observed lower odds of adherence among patients with CLL/SLL residing in the southern United States. Among commercial and Medicare patients taking chronic medications, residence in the southern United States has been found to be associated with lower odds of medication adherence. ${ }^{44,45}$

In our study, we found that adherent patients had reduced risk of all-cause health care utilization and lower all-cause medical costs and is in line with previous literature indicating reduced utilization and costs with increased adherence. ${ }^{8,9}$ Promoting better adherence to oral oncolytics may help reduce future health resource and related financial burden on the patient. Conversely, adherent patients experienced higher prescription costs. The costs of cancer therapies have been increas- ing over the years, and adherent patients might incur more costs due to medication-taking behavior. ${ }^{46}$ Additionally, it can be hypothesized that a positive medication-taking behavior for oral oncolytics may influence their medication- taking behaviors for concurrent medications, leading to higher incurred prescription costs.

\section{Limitations}

This study was limited in several ways. First, we used a convenience sample of patients with commercial or Medicare supplemental insurance; thus, the results might not be generalizable to a broader, more heterogeneous population, such as Medicare Advantage and fee-for-service populations, which might offer a better representation of patients with these cancers, as majority cases are diagnosed in and after age 60 years. Interestingly, our MM and CLL/SLL cohorts were younger than the median age of diagnosis for these cancers. However, previous literature has used administrative claims like MarketScan to study outcomes in these cancers. $3,19,20,47-49$

Second, we used prescription fills to assess medication adherence, which do not corroborate whether patients actually took the oncolytic; however, such indirect measures have been extensively used in epidemiologic research as a reliable medication adherence metric.

Third, we used administrative claims data, which have inherent limitations such as potential miscoding and missing data, lack of data on cancer severity/staging, year of diagnosis/ length of cancer, number of prior lines of therapy, and reasons for medication nonadherence/discontinuation.

Fourth, we studied patients who had continuous enrollment for 12 months following oncolytic initiation, thus, our results might not be applicable to those who might die within a year of initiation. However, with improved survival rates with oral oncolytics, it might be unlikely that patients will die within a year of oral therapy initiation.

Fifth, we found mixed results on factors influencing adherence such as age and insurance type. However, previous studies on cancers have also found inconsistent results on various factors affecting adherence depending on the characteristics of the data source and patients being studied.

Finally, we wanted to assess adherence to recently approved multiple myeloma therapies ixazomib and panobinostat over a longer period of time as a part of the main analysis but could not because they were only approved in 2015, and hence, there was a lack of sufficient follow-up. However, we assessed adherence to these therapies as a supplementary analysis over a shorter time period.

\section{Conclusions}

Adherence to oral oncolytics for MM and CLL/SLL was low. Adherence to oral oncolytics for hematologic cancers may be influenced by patient-, treatment-, and health system-related 


\section{Medication Adherence, Health Care Utilization, and Costs Among Patients Initiating Oral Oncolytics for Multiple Myeloma or Chronic Lymphocytic Leukemia/Small Lymphocytic Lymphoma}

factors, such as increased outpatient utilization. Adherence to oral oncolytics may reduce health care utilization and costs and eventually reduce the economic burden faced by the patients, health care system and the payer. Health care professionals should consider the identified predictors when promoting adherence to oral oncolytics for hematologic cancers. These factors may help guide health system efforts such as pharmacist-/physician-based counseling, medication reminders from physician's offices, and incentives for proper medication taking, lowering health care utilization and costs, and, eventually, improving patient outcomes in patients with MM or CLL/SLL using oral oncolytics.

\section{Authors}

ANKUR A. DASHPUTRE, MS, Institute for Health Outcomes and Policy, College of Graduate Health Sciences, University of Tennessee Health Science Center, Memphis; KATIE S. GATWOOD, PharmD, Vanderbilt University Medical Center, Nashville, Tennessee; and JUSTIN GATWOOD, PhD, College of Pharmacy, University of Tennessee Health Science Center, Nashville.

AUTHOR CORRESPONDENCE: Ankur A. Dashputre, MS, Institute for Health Outcomes and Policy, College of Graduate Health Sciences, University of Tennessee Health Science Center, 956 Court Ave., Coleman B212, Memphis, TN 38163. Tel.: 901.448.5358; E-mail: adashput@uthsc.edu.
7. Furman RR, Sharman JP, Coutre SE, et al. Idelalisib and rituximab in relapsed chronic lymphocytic leukemia. N Engl J Med. 2014;370(11):997-1007.

8. Greer JA, Amoyal N, Nisotel L, et al. A systematic review of adherence to oral antineoplastic therapies. Oncologist. 2016;21(3):354-76.

9. Cutler RL, Fernandez-Llimos F, Frommer M, Benrimoj C, GarciaCardenas V. Economic impact of medication non-adherence by disease groups: a systematic review. BMJ Open. 2018;8(1):e016982

10. Winn AN, Keating NL, Dusetzina SB. Factors associated with tyrosine kinase inhibitor initiation and adherence among Medicare beneficiaries with chronic myeloid leukemia. J Clin Oncol. 2016;34(36):4323-28.

11. Haque R, Shi J, Chung J, et al. Medication adherence, molecular monitoring, and clinical outcomes in patients with chronic myelogenous leukemia in a large HMO. J Am Pharm Assoc (2003). 2017;57(3):303-10 e302.

12. Rychter A, Jerzmanowski P, Holub A, et al. Treatment adherence in chronic myeloid leukaemia patients receiving tyrosine kinase inhibitors. Med Oncol. 2017;34(6):104

13. Dusetzina SB, Winn AN, Abel GA, Huskamp HA, Keating NL. Cost sharing and adherence to tyrosine kinase inhibitors for patients with chronic myeloid leukemia. J Clin Oncol. 2014;32(4):306-11.

14. Shen C, Zhao B, Liu L, Shih YT. Adherence to tyrosine kinase inhibitors among Medicare Part D beneficiaries with chronic myeloid leukemia. Cancer 2018;124(2):364-73

15. Santoleri F, Lasala R, Ranucci E, et al. Medication adherence to tyrosine kinase inhibitors: 2-year analysis of medication adherence to imatinib treatment for chronic myeloid leukemia and correlation with the depth of molecular response. Acta Haematol. 2016;136(1):45-51.

16. Latremouille-Viau D, Guerin A, Gagnon-Sanschagrin P, Dea K, Cohen BG, Joseph GJ. Health care resource utilization and costs in patients with chronic myeloid leukemia with better adherence to tyrosine kinase inhibitors and increased molecular monitoring frequency. J Manag Care Spec Pharm. 2017;23(2):214-24. Available at: https://www.jmcp.org/doi/10.18553/ jmcp.2017.23.2.214

17. Ganesan P, Sagar TG, Dubashi B, et al. Nonadherence to imatinib adversely affects event free survival in chronic phase chronic myeloid leukemia. Am J Hematol. 2011;86(6):471-74.

18. Anderson KR, Chambers CR, Lam N, et al. Medication adherence among adults prescribed imatinib, dasatinib, or nilotinib for the treatment of chronic myeloid leukemia. J Oncol Pharm Pract. 2015;21(1):19-25.

19. Chari A, Bhor M, Eldjerou L, et al. Treatment patterns and medication adherence among patients diagnosed with multiple myeloma and treated with panobinostat. Future Oncol. 2018;14(21):2149-60.

20. Song X, Cong Z, Wilson K. Real-world treatment patterns, comorbidities, and disease-related complications in patients with multiple myeloma in the United States. Curr Med Res Opin. 2016;32(1):95-103.

21. Cransac A, Aho S, Chretien ML, Giroud M, Caillot D, Boulin M. Adherence to immunomodulatory drugs in patients with multiple myeloma. PLoS One. 2019;14(3):e0214446.

22. Teitelbaum A, Ba-Mancini A, Huang H, Henk HJ. Health care costs and resource utilization, including patient burden, associated with novel-agentbased treatment versus other therapies for multiple myeloma: findings using real-world claims data. Oncologist. 2013;18(1):37-45.

23. Lee C, Grigorian M, Nolan R, Binder G, Rice G. A retrospective study of direct cost to patients associated with the use of oral oncology medications for the treatment of multiple myeloma. J Med Econ. 2016;19(4):397-402.

24. Kumar SK, Callander NS, Alsina M, et al. NCCN guidelines insights: multiple myeloma, version 3.2018. J Natl Compr Canc Netw. 2018;16(1):11-20.

25. Rajkumar SV. Value and cost of myeloma therapy. Am Soc Clin Oncol Educ Book. 2018;38:662-66 


\section{Medication Adherence, Health Care Utilization, and Costs Among Patients Initiating Oral Oncolytics for Multiple Myeloma or Chronic Lymphocytic Leukemia/Small Lymphocytic Lymphoma}

26. Carlson JJ, Guzauskas GF, Chapman RH, et al. Cost-effectiveness of drugs to treat relapsed/refractory multiple myeloma in the United States. J Manag Care Spec Pharm. 2018;24(1):29-38. Available at: https://www.jmcp. org/doi/10.18553/jmcp.2018.24.1.29.

27. National Cancer Institute. NCI comorbidity index overview. May 23, 2019. Available at: https://healthcaredelivery.cancer.gov/seermedicare/considerations/comorbidity.html. Accessed January 6, 2020

28. Pharmacy Quality Alliance. PQA Measure overview. 2019. Available at: https://www.pqaalliance.org/assets/Measures/2019_PQA_Measure_ Overview.pdf. Accessed January 21, 2020.

29. Wu EQ, Johnson S, Beaulieu N, et al. Healthcare resource utilization and costs associated with non-adherence to imatinib treatment in chronic myeloid leukemia patients. Curr Med Res Opin. 2010;26(1):61-69.

30. Yood MU, Oliveria SA, Cziraky M, Hirji I, Hamdan M, Davis C. Adherence to treatment with second-line therapies, dasatinib and nilotinib, in patients with chronic myeloid leukemia. Curr Med Res Opin. 2012;28(2):213-19.

31. Ward MA, Fang G, Richards KL, et al. Comparative evaluation of patients newly initiating first-generation versus second-generation tyrosine kinase inhibitors for chronic myeloid leukemia and medication adherence, health services utilization, and healthcare costs. Curr Med Res Opin. 2015;31(2):289-97.

32. Smith BD, Liu J, Latremouille-Viau D, Guerin A, Fernandez D, Chen L. Treatment patterns, overall survival, healthcare resource use and costs in elderly Medicare beneficiaries with chronic myeloid leukemia using secondgeneration tyrosine kinase inhibitors as second-line therapy. Curr Med Res Opin. 2016;32(5):817-27.

33. U.S. Bureau of Labor Statistics. Top picks. CPI for all urban consumers (CPI-U). Available at: https://data.bls.gov/cgi-bin/surveymost?cu. Accessed January 21, 2020

34. International Society for Pharmacoeconomics and Outcomes Research Measuring multiple medication adherence: which measure when? November 2, 2016. Available at: https://www.ispor.org/docs/default-source/presentations/980.pdf?sfvrsn=bf652b87_1. Accessed January 6, 2020.

35. Ruddy K, Mayer E, Partridge A. Patient adherence and persistence with oral anticancer treatment. CA Cancer J Clin. 2009;59(1):56-66.

36. Puts MT, Tu HA, Tourangeau A, et al. Factors influencing adherence to cancer treatment in older adults with cancer: a systematic review. Ann Oncol. 2014;25(3):564-77.
37. Piccirillo JF, Vlahiotis A, Barrett LB, Flood KL, Spitznagel EL, Steyerberg EW. The changing prevalence of comorbidity across the age spectrum. Crit Rev Oncol Hematol. 2008;67(2):124-32

38. Choudhry NK, Fischer MA, Avorn J, et al. The implications of therapeutic complexity on adherence to cardiovascular medications. Arch Intern Med. 2011:171(9):814-22

39. Heckman BW, Mathew AR, Carpenter MJ. Treatment burden and treatment fatigue as barriers to health. Curr Opin Psychol. 2015;5:31-36.

40. Ingersoll KS, Cohen J. The impact of medication regimen factors on adherence to chronic treatment: a review of literature. J Behav Med. 2008;31(3):213-24.

41. Saini SD, Schoenfeld P, Kaulback K, Dubinsky MC. Effect of medication dosing frequency on adherence in chronic diseases. Am J Manag Care. 2009;15(6):e22-33.

42. Pantuzza LL, Ceccato M, Silveira MR, Junqueira LMR, Reis AMM Association between medication regimen complexity and pharmacotherapy adherence: a systematic review. Eur J Clin Pharmacol. 2017;73(11):1475-89.

43. Partridge AH, Avorn J, Wang PS, Winer EP. Adherence to therapy with oral antineoplastic agents. J Natl Cancer Inst. 2002;94(9):652-61.

44. Couto JE, Panchal JM, Lal LS, et al. Geographic variation in medication adherence in commercial and Medicare part D populations. J Manag Care Spec Pharm. 2014;20(8):834-842. Available at: https://www.jmcp.org/ doi/10.18553/jmcp.2015.21.12.1195.

45. Tan E, Yang W, Pang B, Dai M, Loh FE, Hogan P. Geographic variation in antidiabetic agent adherence and glycemic control among patients with type 2 diabetes. J Manag Care Spec Pharm. 2015;21(12):1195-202. Available at: https://www.jmcp.org/doi/10.18553/jmcp.2015.21.12.1195.

46. Shih YC, Smieliauskas F, Geynisman DM, Kelly RJ, Smith TJ. Trends in the cost and use of targeted cancer therapies for the privately insured nonelderly: 2001 to 2011. J Clin Oncol. 2015;33(19):2190-26.

47. Hagiwara M, Panjabi S, Sharma A, Delea TE. Healthcare utilization and costs among relapsed or refractory multiple myeloma patients on carfilzomib or pomalidomide as monotherapy or in combination with dexamethasone. J Med Econ. 2019;22(8):818-29.

48. Maiese EM, Evans KA, Chu BC, Irwin DE. Temporal trends in survival and healthcare costs in patients with multiple myeloma in the United States. Am Health Drug Benefits. 2018;11(1):39-46.

49. Reyes C, Engel-Nitz NM, DaCosta Byfield S, et al. Cost of disease progression in patients with chronic lymphocytic leukemia, acute myeloid leukemia, and non-Hodgkin's lymphoma. Oncologist. 2019;24(9):1219-28. 
Medication Adherence, Health Care Utilization, and Costs Among Patients Initiating Oral Oncolytics for Multiple Myeloma or Chronic Lymphocytic Leukemia/Small Lymphocytic Lymphoma

\section{APPENDIX A Patient Selection Flow Criteria}

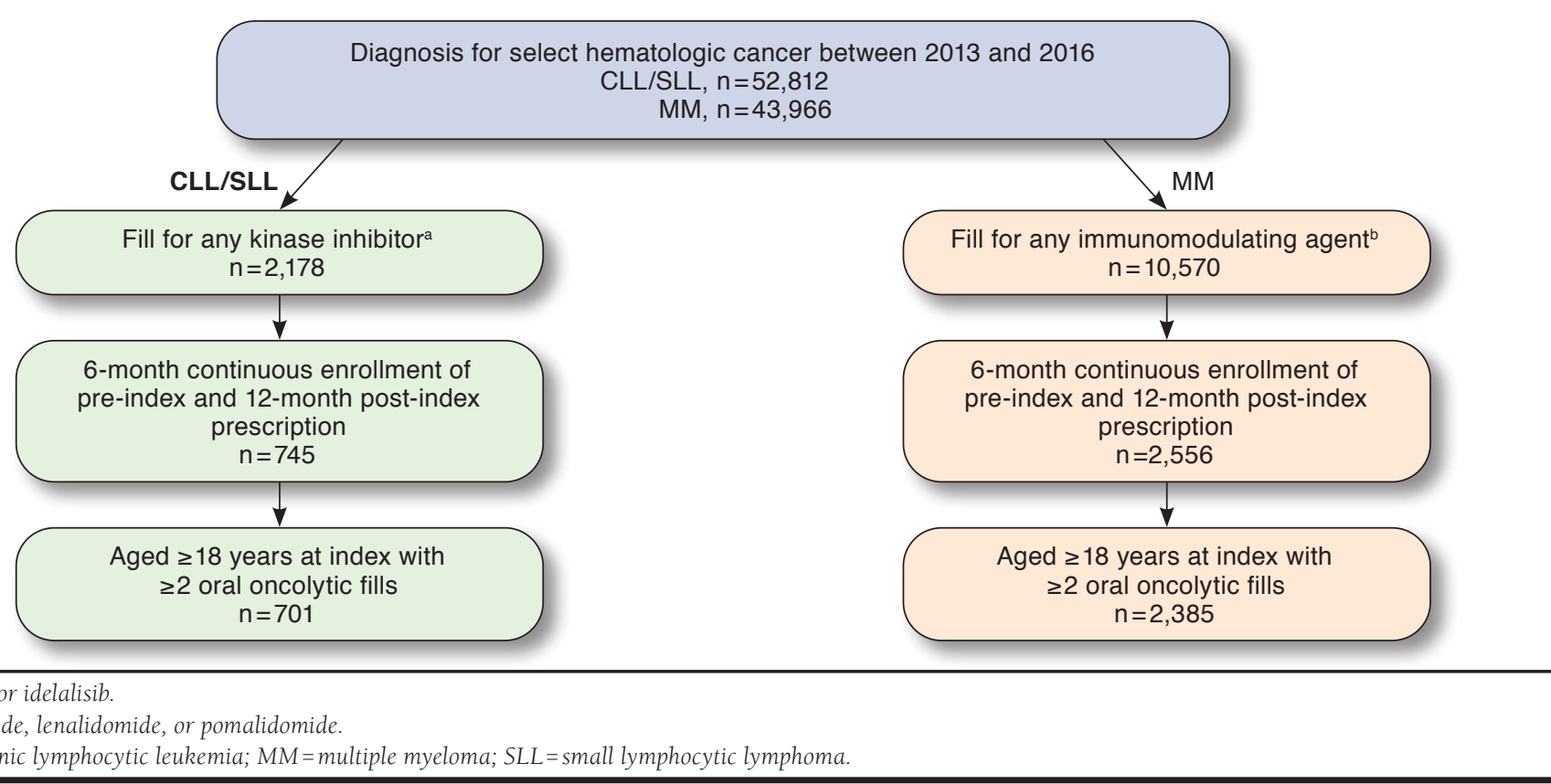


Medication Adherence, Health Care Utilization, and Costs Among Patients Initiating Oral Oncolytics for Multiple Myeloma or Chronic Lymphocytic Leukemia/Small Lymphocytic Lymphoma

\section{APPENDIX B Factors Associated with Adherence to Oral Oncolytics (Sensitivity Analyses)}

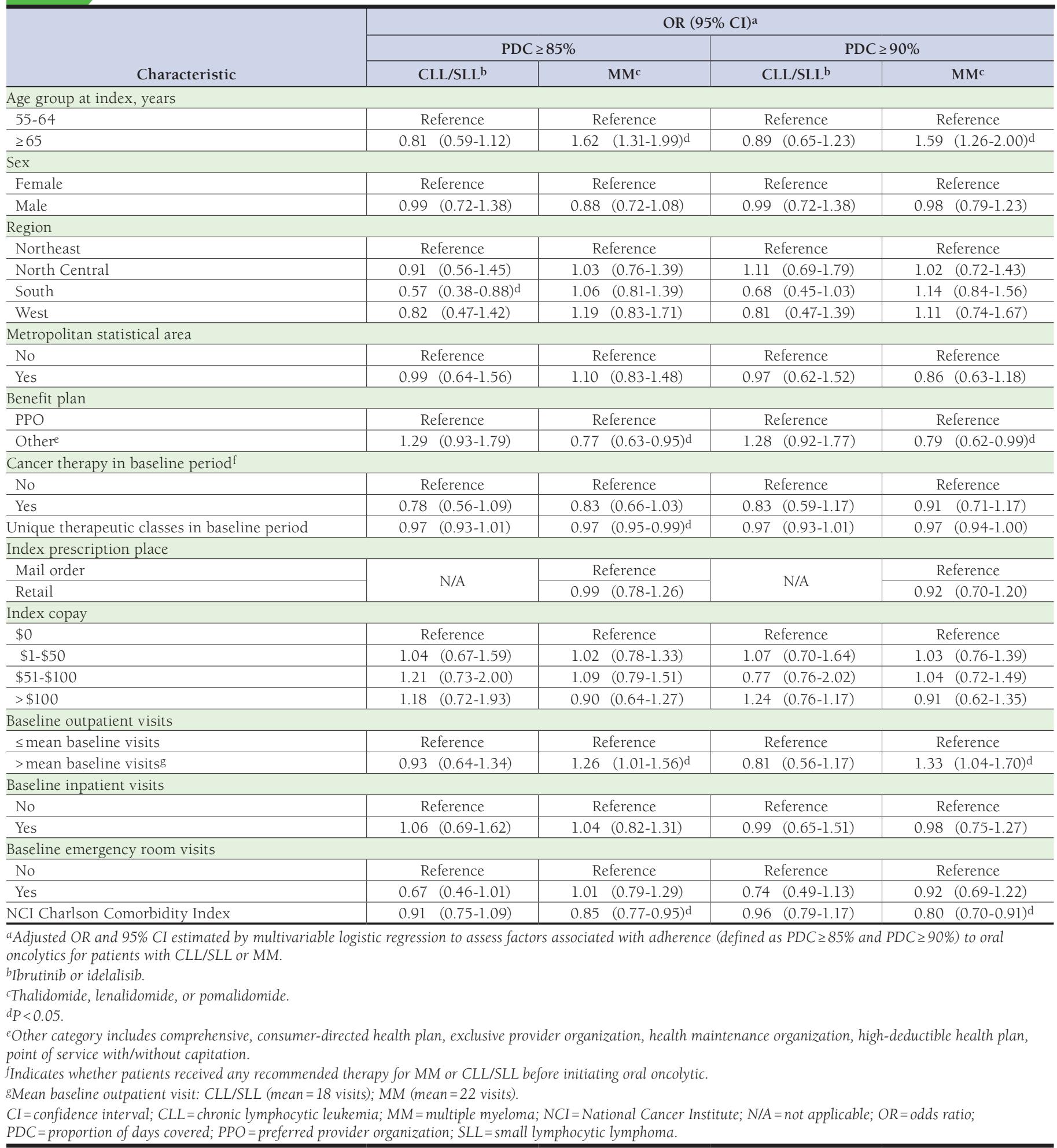

